\title{
Temperature and magnetic-field driven dynamics in artificial magnetic square ice
}

\author{
Sophie A. Morley ${ }^{a}$, Aaron Stein ${ }^{b}$, Mark C. Rosamond ${ }^{c}$, Diego Alba Venero ${ }^{d}$, Aleš Hrabec ${ }^{a}$, \\ Philippa M. Shepley ${ }^{a}$, Mi-Young Im ${ }^{e, f}$, Peter Fischer ${ }^{e, g}$, Matthew T. Bryan ${ }^{h}$, Dan A. Allwood ${ }^{h}$ \\ , Paul Steadman ${ }^{i}$, Sean Langridge ${ }^{d}$, and Christopher H. Marrows ${ }^{a}$ \\ ${ }^{a}$ School of Physics \& Astronomy, University of Leeds, Leeds LS2 9JT, United Kingdom; \\ ${ }^{b}$ Center for Functional Nanomaterials, Brookhaven National Laboratory, \\ Upton NY 11793, USA; \\ ${ }^{c}$ School Electronic and Electrical Engineering, University of Leeds, \\ Leeds LS2 9JT, United Kingdom; \\ ${ }^{d}$ ISIS, STFC Rutherford Appleton Laboratory, \\ Didcot, Oxon. OX11 0QX, United Kingdom; \\ ${ }^{e}$ Center for X-ray Optics, Lawrence Berkeley National Laboratory, \\ 1 Cyclotron Road, Berkeley, CA 94720, USA; \\ ${ }^{f}$ Daegu Gyeongbuk Institute of Science and Technology, Daegu 711-873, Korea; \\ ${ }^{g}$ Department of Physics, University of California, Santa Cruz, California 94056, USA; \\ ${ }^{h}$ Department of Materials Science and Engineering, University of Sheffield, \\ Mappin Street, Sheffield S1 3JD, United Kingdom; \\ ${ }^{i}$ Diamond Light Source, Didcot, Oxon. OX11 0DE, United Kingdom
}

\begin{abstract}
Artificial spin ices are often spoken of as being realisations of some of the celebrated vertex models of statistical mechanics, where the exact microstate of the system can be imaged using advanced magnetic microscopy methods. The fact that a stable image can be formed means that the system is in fact athermal and not undergoing the usual finite-temperature fluctuations of a statistical mechanical system. In this paper we report on the preparation of artificial spin ices with islands that are thermally fluctuating due to their very small size. The relaxation rate of these islands was determined using variable frequency focussed magneto-optic Kerr measurements. We performed magnetic imaging of artificial spin ice under varied temperature and magnetic field using X-ray transmission microscopy which uses X-ray magnetic circular dichroism to generate magnetic contrast. We have developed an on-membrane heater in order to apply temperatures in excess of $700 \mathrm{~K}$ and have shown increased dynamics due to higher temperature. Due to the 'photon-in, photon-out' method employed here, it is the first report where it is possible to image the microstates of an ASI system under the simultaneous application of temperature and magnetic field, enabling the determination of relaxation rates, coercivties, and the analysis of vertex population during reversal.
\end{abstract}

Keywords: Artificial spin ice, vertex model, nanomagnetism, magnetic soft X-ray transmission microscopy

\section{INTRODUCTION}

Recently a new species of magnetic metamaterials has emerged, known as artificial spin ices (ASIs). ${ }^{1-3}$ They consist of a lithographically patterned nanoscale magnetic islands arranged in a 2-dimensional array such that the islands are interacting through their stray fields and thereby exhibit magnetic frustration phenomena. An early report of such a system was made by Wang et al., ${ }^{4}$ who studied magnetic square ice. A scanning electron

Further author information: (Send correspondence to C.H.M.)

C.H.M.: E-mail: c.h.marrows@leeds.ac.uk, Telephone: +44 1133433780

M.T.B.Present address: Department of Cardiovascular Science, University of Sheffield, Sheffield, S10 2RX.

Spintronics VIII, edited by Henri-Jean Drouhin, Jean-Eric Wegrowe, Manijeh Razeghi, Proc. of SPIE Vol. 9551, 95511Q · @ 2015 SPIE · CCC code: 0277-786X/15/\$18 - doi: 10.1117/12.2189320 


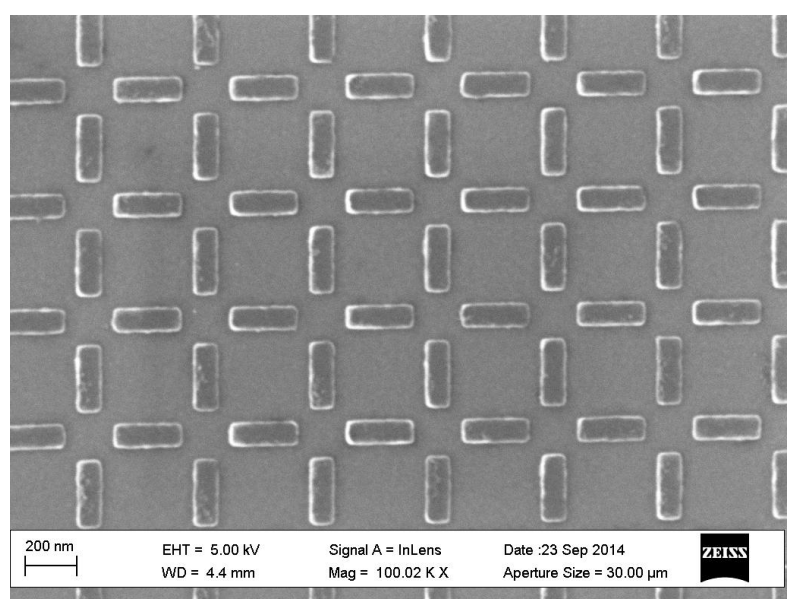

Figure 1. Scanning electron micrograph of an artificial magnetic square ice array, formed from a pattern of Permalloy islands. The nanomagnets are sized so as to be single domain macrospins and shaped so as to to give Ising-like character to those macrospins. The islands are arranged along the edges of a square lattice, leading to vertex-level frustration.

micrograph of an example of such a system is shown in Fig. 1. The size of the islands is small enough that they act as single domain Stoner-Wohlfarth particles, ${ }^{5}$ and so can be modelled as possessing a macrospin. The shape of the islands is designed so as to confine that spin to point along one of a pair of easy directions in the array plane and so are Ising-like. The name 'artificial spin ices' arises from the fact that they were originally designed to provide nanoscale analogs to the "naturally-occurring" 3 -dimensional rare earth pyrochlores. ${ }^{6}$ These in turn are magnetic analogs of the most common form of water ice (Ice $\left.I_{h}\right)$, in which a closely related tetrahedral lattice structure leads to geometrical frustration in the position of protons, giving rise to an extensive degeneracy and residual entropy. ${ }^{7}$ The pyrochlore spin ices show exactly the same value of residual entropy, ${ }^{8}$ and their excitations can be treated as magnetic monopoles connected by Dirac strings of flipped rare earth moments. ${ }^{9}$

Both Ice $\mathrm{I}_{\mathrm{h}}$ and the pyrochlore spin ices adopt a two-in/two-out ice rule at each 3-dimensional tetrahedral vertex. In water ice this is known as the Bernal-Fowler ice rule. ${ }^{10}$ In the square ices the vertices are of lower, square symmetry due to their 2-dimensional nature, as shown in Fig. 2(a). The magnetostatic coupling between each pair of elements would prefer them to adopt a head-to-tail configuration on energetic grounds. Since this is not possible for all six pairwise interactions simultaneously, this vertex possesses geometrical frustration. There are $2^{4}=16$ possible arrangements of the four Ising-like moments, which are depicted in Fig. 2(b). These can
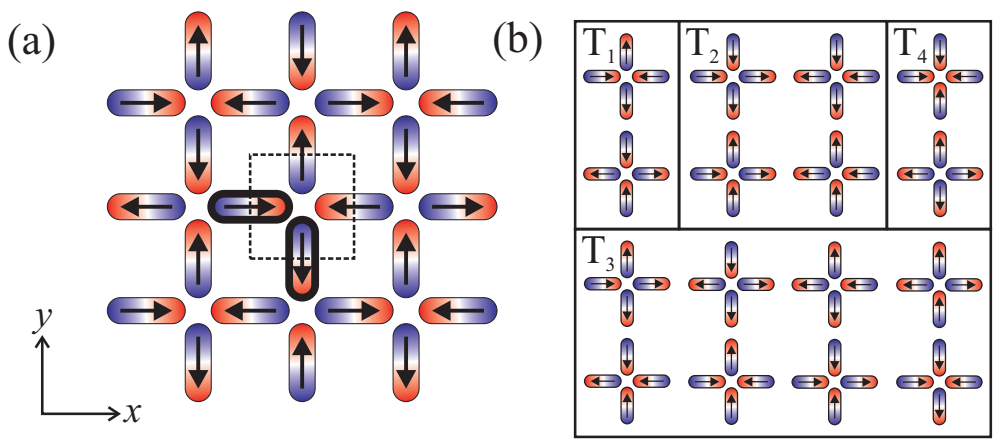

Figure 2. Square ice vertex geometry. (a) The square ice ground state. Each magnetic nano-element possesses an Ising-like moment, as indicated by arrows. The dashed box indicates a structural unit cell (with lattice constant $a$ ) containing a single vertex, which possesses an area of $a^{2}$. An example of a pair of $1^{\text {st }}$ nearest neighbour moments is emboldened. Cartesian axes are also defined with reference to the sample frame. (b) The 16 vertex model of square ice. Vertices can be arranged into four types, labelled $\mathrm{T}_{i}$, in order of increasing energy, with $i=1$ to 4 . 
be subdivided into four types, which are conventionally labelled Type 1-4 in order of increasing energy. Types 1 and 2 obey the two-in/two-out ice rule. The two configurations that form Type 1 have their energy lowered with respect to Type 2 by the lowered symmetry of the square vertex. These six vertex configurations possess no net magnetic charge: the poles at the centre of the vertex all cancel. The Type 3 configurations are either three-in/one-out or one-in/three-out and possess a net magnetic charge, and so correspond to the monopoles of Castelnovo et al.,$^{9}$ although the reduced symmetry of the square lattice means that the monopoles and connecting strings are of the Nambu type in square ASI systems. ${ }^{11}$ The two Type 4 configurations are all-in or all-out and are thus very energetic, possessing a double charge.

It has become a commonplace that ASIs are realisations of the "toy" square ice models of statistical mechanics that were solved long ago by researchers such as $\mathrm{Wu}^{12}$ and Lieb. ${ }^{13}$ Nevertheless, temperature $T$ plays a key role in statistical mechanics, but is irrelevant to most previously studied ASIs. This is because the relevant energy scales for the interisland coupling and island reversal barriers are of the order of $10^{4}-10^{5} \mathrm{~K}$ for typical island sizes and spacings, such as those shown in Fig. 1, and so these are athermal systems. Whilst it is possible to describe these within an effective thermodynamics, ${ }^{14-17}$ the energy of the system does not extrapolate to the ground state. ${ }^{18}$ Achieving true thermalisation has thus been an important goal of much recent ASI research.

This was achieved by Morgan et al. using a one-shot equilibration method where the islands have a small enough volume during the early stages of growth to be thermally active, but then the thermalised state is frozen in as the elements continue to grow. ${ }^{19}$ The macrospin ice system has been shown to melt if it is heated close to the Curie point of the underlying microspin system. ${ }^{20}$ Developing this concept, it has also been shown that it is possible to repeatedly thermalise an ASI system by using high temperatures to heat the sample in order to access dynamics, cool, then image the arrested state. ${ }^{21,22}$ Nevertheless, it is desirable to see the dynamics as they happen in real time. More recently studies have been carried out on very thin samples using x-ray photoemission electron microscopy (PEEM), and have shown real time thermal spin ice in both the kagomé23,24 and square ice geometries. ${ }^{25}$ That work provided the first direct view of thermally active systems. However, since PEEM requires photoelectrons to be extracted from the sample to form the image, magnetic fields cannot be applied during imaging, which means that the sample is always in a remanent state. Here we use magnetic X-ray transmission microscopy (M-XTM), which is a method that only relies on photons, to image thermally activated dynamics in a square artificial spin ice under the application of a magnetic field. M-XTM experiments require the sample to be fabricated on an X-ray transparent membrane. We have also developed an on-membrane microheater and thermometer system so that it was also been possible to explore the effect of both varied temperature and applied field with direct imaging. In order to achieve thermally activated dynamics it is necessary to prepare ASI systems with smaller elements than in the past, since the energy barrier that provides thermal stability is given by $K V$, where $K$ is the anisotropy constant provided by the island shape and $V$ is the volume of an individual island. Thus $K=\frac{1}{2} \mu_{0} D M^{2}$, where $M$ is the magnetization of the material from which the island is fabricated and $D$ is a suitable demagnetizing factor. It can be seen that small volumes are needed to prevent complete freezing of the macrospin system. When fully melted, the macrospins are superparamagnetic and fluctuate at a rate $f_{0}$ that is typically 1-10 GHz, far too fast for single-shot magnetic imaging techniques. (Such fast processes may be imaged stroboscopically if they are entirely repeatable, the exact opposite of the random thermal fluctuations that we study here.) The relaxation rate $1 / \tau$ of a particular island is given by the Néel-Arrhenius expression

$$
\frac{1}{\tau}=f_{0} \exp \left(-\frac{\Delta E}{k_{\mathrm{B}} T}\right)
$$

where $k_{\mathrm{B}}$ is the Boltzmann constant and $\Delta E=K V+E_{\mathrm{c}}$. Here we define $E_{\mathrm{c}}$ as the modification to the energy barrier arising from the magnetostatic coupling to neighboring islands, which may be positive (stabilizing against reversal) or negative (destabilizing), depending on the exact configuration of the moments of those islands. We have used the magneto-optic Kerr effect (MOKE) to probe the hysteresis of ASI systems at different frequencies and island sizes in order to show how to tune our ASI systems into a size regime where $\tau$ is a few seconds, the typical image acquisition time in a full-field transmission soft X-ray microscopy experiment. We show that the energy barrier to reversal may be estimated from measured island flip rates and tuned with field. We report on vertex population statistics during thermally activated reversal of ASI arrays at different temperatures, where a preference for monopole-antimonopole pair production over Nambu string extension was discovered. Thus we use two optical methods, involving visible and soft X-ray photons, to study thermal activity in square ASIs. 


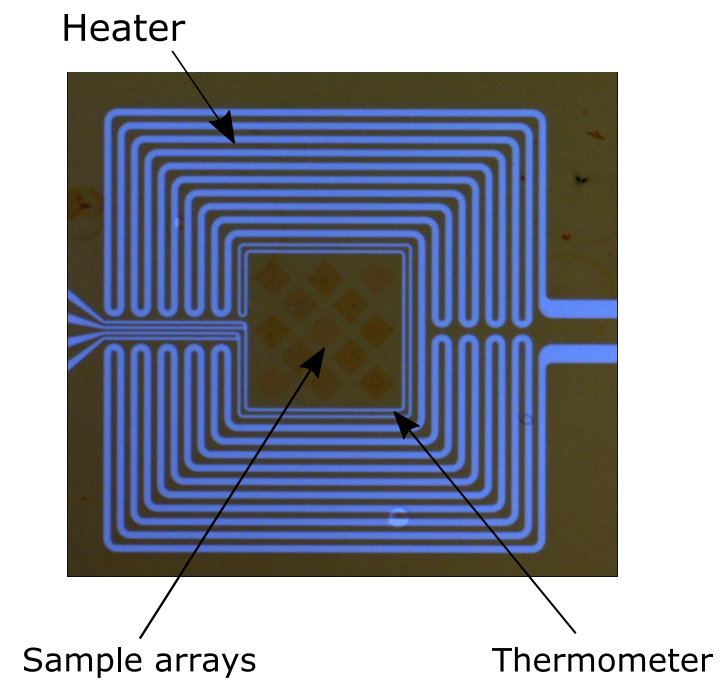

Figure 3. Optical micrograph of the heater and thermometer design for the on-membrane sample heating. The wiring is inductively counterwound in order to avoid magnetic fields at the sample position.

\section{FABRICATION OF ARTIFICIAL SPIN ICES}

The ASIs used in these experiments were fabricated by electron beam lithography (EBL) and sputter deposition of magnetic metals. The ASIs used in the MOKE experiments were made using $10 \mathrm{~nm}$ thick Permalloy $\left(\mathrm{Ni}_{81} \mathrm{Fe}_{19}\right)$ films with a $3 \mathrm{~nm}$ aluminium cap and standard liftoff methods. For the M-XTM experiments, a magnetic film was deposited by sputtering using an alloyed $\mathrm{Co}_{68} \mathrm{Fe}_{22} \mathrm{~B}_{10}$ target on to a $\mathrm{Si}_{3} \mathrm{~N}_{4}$ membrane to form a film that was $10 \mathrm{~nm}$ thick. EBL was used to expose the ASI array, of nanomagnets with lateral dimensions of $80 \times 250 \mathrm{~nm}$, with a lattice spacing of $500 \mathrm{~nm}$. A hard mask was then deposited using electron beam evaporation and lift-off after which a Ar ion-beam milling removed the unpatterned CoFeB and mask, to leave CoFeB nanostructures with $10 \mathrm{~nm}$ thickness. A microheater and thermometer were then patterned using EBL around the samples in order to control the temperature, as shown in Fig. 3. Pt was used as the evaporated material for the resistive heating and thermometry, since its resistivity has a linear dependence on temperature. The thermometer was calibrated by immersing the membrane in a bath of deionised water and measuring the four-point thermometer resistance as the temperature was raised after the microscopy experiment was completed. The maximum temperature we applied during the experiment was $705 \mathrm{~K}$, obtained with a heater current, $I_{\mathrm{h}}=5 \mathrm{~mA}$.

\section{FOCUSSED MAGNETO-OPTIC KERR EFFECT MEASUREMENTS}

The magneto-optic Kerr effect (MOKE) is a convenient way to measure small magnetic structures. Here we used focussed $\mathrm{MOKE}^{26}$ (fMOKE) to measure the coercive field $H_{\mathrm{c}}$ of Permalloy ASI arrays as a function of time $t$ taken to complete a hysteresis loop. The need to focus the the laser spot to $\sim 5 \mu \mathrm{m}$ diameter was due to the micron scale of the artificial spin ice arrays. The fMOKE setup was similar to that described by Allwood et al. ${ }^{26}$

The measurements were made under an applied magnetic field that oscillated in time with a sinusoidal wave profile. The period of the oscillation is defined as the time period $t$. Some example hysteresis loops, each the average of many loops acquired during the field oscillation, are shown in Fig. 4. In this case the Permalloy islands have lateral size $18 \mathrm{~nm} \times 56 \mathrm{~nm}$ and are $10 \mathrm{~nm}$ thick. When cycled at the quickest rate, which in this case is for $t=0.04 \mathrm{~s}$, substantial hysteresis is present. This is gradually lost as the period of the field oscillation is increased. When the hysteresis loop is traversed at a slow rate, so that $t=10.0 \mathrm{~s}$, the coercivity has almost vanished. This is because the nanoislands are able to thermally relax over their energy barriers whilst the measurement is taking place, they are not able to do this when the loop is traversed quickly and so in that case coercivity is maintained.

These measurements were performed for several different islands sizes and the coercive field $H_{\mathrm{c}}$ was determined for each size and field oscillation period $t$. A summary of the data is shown in Fig. 5. For larger island sizes-viz. 


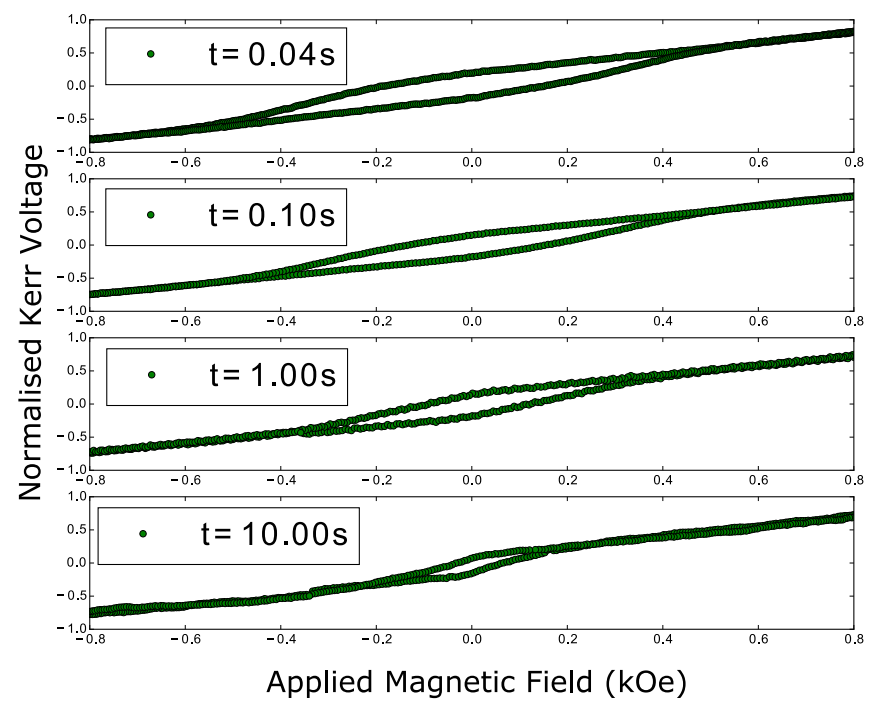

Figure 4. Hysteresis loops of an ASI array with a lateral island size of $18 \mathrm{~nm} \times 56 \mathrm{~nm}$ by fMOKE. The islands were fabricated from $10 \mathrm{~nm}$ thick Permalloy film. Each loop is an average of many measurements made under a field oscillating with period $t$.

those larger than about $30 \mathrm{~nm}$ wide-a significant coercivity of hundred of Oe is seen across the whole range of measurement timescales $10 \mathrm{~ms} \lesssim t \lesssim 10 \mathrm{~s}$. The hysteresis loop is never traversed slowly enough to permit significant relaxation. For the smallest islands ( $8 \mathrm{~nm}$ wide), there is never any significant coercivity at any timescale. In this case, the loop is never traversed quickly enough to prevent relaxation. The $18 \mathrm{~nm}$ wide islands, for which data were shown in Fig. 4, form an interesting intermediate case, where the coercivity collapses in the window of timescales to which we have experimental access. It is islands with values of $V$ such as these that have relaxation times of the order of the loop measurement timescale where the coercivity collapses, i.e. $\tau \sim t$. Thus, this method provides a convenient means for assessing the likely rates at which the magnetic moments in the islands of a given size will fluctuate and thus finding samples that match experimental timescales for measurements where experimental time is scarce, such as at synchrotron sources.

According to Bean and Livingston, ${ }^{27}$ the energy barrier $\Delta E$ is modified by a factor which depends on the field $H$ that is applied,

$$
\Delta E=K V\left(1-\frac{H}{H_{0}}\right)^{n}
$$

where $H_{0}$ is a characteristic field, and the exponent $n$ depends on the details of how the field is applied. If $H$ is applied exactly along the easy axis, as is the case here, $n=2$. (In fact, only half the islands experience the field along their easy axis. The other half experience it exactly along their hard axis, and so do not contribute to any observed coercivity, and may be neglected in this analysis.) Combining Eq. 2 with Eq. 1 it is possible to derive the Sharrock equation for the timescale dependence of the dynamic coercive field, ${ }^{28}$

$$
H_{\mathrm{c}}=H_{0}\left\{1-\left[\left(\frac{k_{\mathrm{B}} T}{K V}\right) \ln \left(\frac{f_{0} t}{\ln 2}\right)\right]^{\frac{1}{n}}\right\},
$$

where we can identify $H_{0}$ as the switching field in the absence of thermal assistance, we assume $f_{0}=10 \mathrm{GHz}$, and the shape anisotropy $K=15.9 \mathrm{~kJ} / \mathrm{m}^{3}$ (where the demagnetisation factor $D$ can be calculated as 0.04 if a perfect cuboid is assumed. ${ }^{29}$ ). In the Stoner-Wohlfarth model, ${ }^{5} H_{0}=2 K / \mu_{0} M$, where $M$ is the magnetization of the material in question. The Sharrock equation fits the data well for the larger islands. The $\ln 2$ term arises from the assumption that the total magnetisation relaxes exponentially towards its new value in the reverse field, 


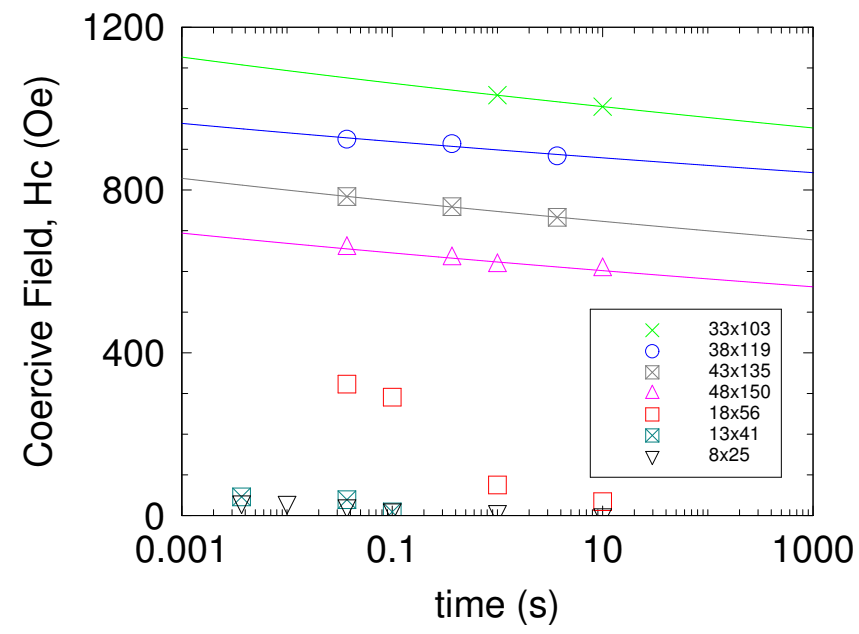

Figure 5. Coercive field measurements by fMOKE for islands of different sizes. Lateral island size is given in the legend in $\mathrm{nm}$ and each array was measured using different times to complete the hysteresis loop. The solid lines are fits of Eq. 3.

with exactly half the particles having flipped their moments at $H_{\mathrm{c}}$. In this simple analysis we have assumed that $K V \gg E_{\mathrm{c}}$.

It can be seen that $H_{0}$ actually rises in this regime as the islands shrink. This is due to the fact that as the islands get smaller there is less opportunity for that magnetization to be non-uniform at their tips, ${ }^{30}$ meaning that their reversal is better described as a coherent rotation of a Stoner-Wohlfarth macrospin. ${ }^{5}$ This is further supported by fact that the the values for the volume returned by the Sharrock fits are actually larger for the smaller islands, equating to a lateral island area $\approx 5000 \mathrm{~nm}^{2}$ for the 33 and $38 \mathrm{~nm}$ islands compared to $\approx 3500$ $\mathrm{nm}^{2}$ for the 43 and $48 \mathrm{~nm}$ islands; suggesting there is a smaller activation area which begins the island reversal for the larger islands. The Sharrock equation cannot be fitted to the data for smallest islands $(<33 \mathrm{~nm})$ not unexpected, since the assumption that $t \gg f_{0}^{-1}$, which forms part of the derivation, does not hold in that case.

\section{MAGNETIC SOFT X-RAY TRANSMISSION MICROSCOPY}

\subsection{Microscope characteristics}

We now turn to experiments where we have performed real-space imaging of this reversal process as it takes place. These measurements were done at the full-field soft X-ray microscope, XM-1, located at beamline 6.1.2 at the Advanced Light Source. This microscope has a spatial resolution of about $25 \mathrm{~nm}$ can be recorded with an exposure time of a few seconds covering a several micrometer large field of view. ${ }^{31}$ All images were taken at the Co $\mathrm{L}_{3}$ edge $(778 \mathrm{eV})$ with circularly polarised X-rays of a fixed helicity. This provided strong X-ray magnetic circular dichroism (XMCD) contrast arising from the high Co content of the CoFeB alloy used: a nanomagnet whose moment is oriented parallel to the X-ray propagation vector will have absorption different from one that has its an anti-parallel, for X-rays at this energy, and this provides the magnetic contrast mechanism. A backthinned, back-illuminated $2048 \times 2048$ pixel CCD camera acts as a detector to form the image, so the absorption is directly measured. Since this method requires differences in magnetisation along the X-ray beam axis, usually the technique is used for samples whose moment lies out-of-the-plane. In order to be sensitive to the in-plane magnetism of our samples it was necessary to tilt the sample $30^{\circ}$ out of the plane. A sample CCD image is shown in Fig. 6(a). Fig. 6(b) shows the magnetic contrast image after dividing two consecutive images with increasing reversal field, darker contrast indicates those islands that have switched their moment orientation during the field step. Here the sample is aligned in such a way that the field is applied in the film plane and along a diagonal of the ASI array, so that all islands have their magnetic easy axis, which is defined by their shape, at $45^{\circ}$ to the field direction. 
(a)

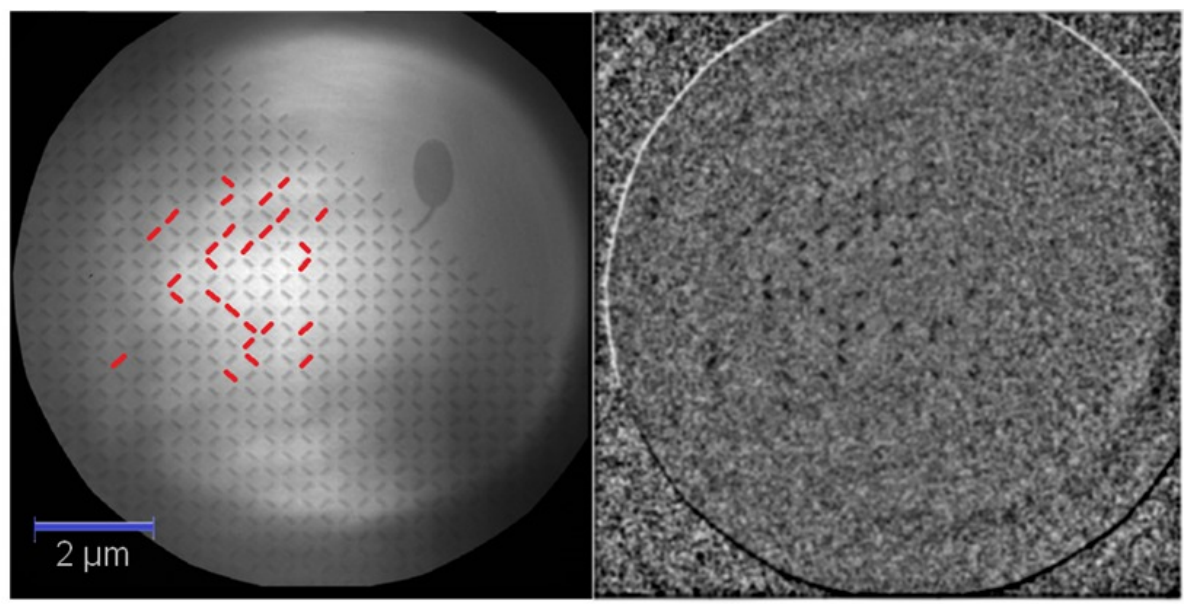

Figure 6. (a) An absorption XTM image of the $80 \times 250 \mathrm{~nm}^{2}$ ASI, exposure time is $1.3 \mathrm{~s}$ with 5 averages. b) The corresponding contrast image showing flipped islands as dark contrast, obtained by dividing consecutive raw absorption images. The islands that have flipped are highlighted in red in panel (a).

\subsection{Thermal behaviour using field-modified barrier height}

All nanomagnets have magnetisation which lies in the plane along their long axis, defined by the shape anisotropy. For these $80 \mathrm{~nm} \times 250 \mathrm{~nm}$ islands, which are $10 \mathrm{~nm}$ thick, the demagnetisation factor $D=0.04$. With the saturation magnetisation of $\mathrm{CoFeB}$ measured by SQUID-vibrating sample magnetometry as $M_{\mathrm{S}} \approx 1.0 \mathrm{MA} / \mathrm{m}$, we find the shape anisotropy $K=25.1 \mathrm{~kJ} / \mathrm{m}^{3}$, yielding an energy barrier to reversal of $K V / k_{\mathrm{B}} \approx 3.6 \times 10^{5} \mathrm{~K}$. At $T=300 \mathrm{~K}$, the ratio of $K V / k_{\mathrm{B}} T \approx 1210$. At this ratio, thermal relaxation will be many orders of magnitude too slow to observe, according Eq. 1.

Hence, in our first experiment we attempted to observe thermally activated flipping events by adjusting the barrier height with magnetic field according to Eq. 2. We measured the sample with $0.6 \mathrm{~s}$ exposure time and 5 averages per image, giving a acquisition time of $3 \mathrm{~s}$. The sample was first saturated in one direction and then an opposite field applied that was $90 \%$ of $H_{\mathrm{c}}$, we then continued taking images for a further $40 \mathrm{~s}$. Shown in Fig. 7 is the cumulative number of flips observed at each waiting time, which grows linearly with time. The slope of a

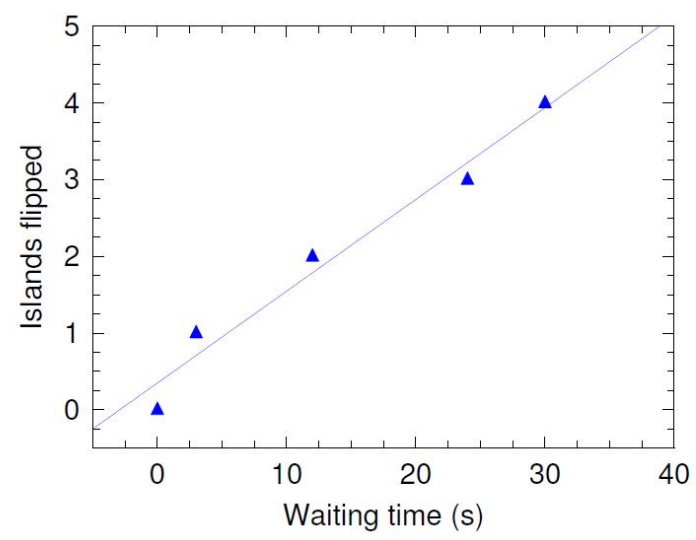

Figure 7. Number of thermally-assisted flipping events as a function of waiting time, after applying a reversal field equivalent to $90 \%$ of the coercivity. The solid line is a straight line best fit to the data, the slope of which gives the average flipping rate $r=0.12 \pm 0.02 \mathrm{~s}^{-1}$. 


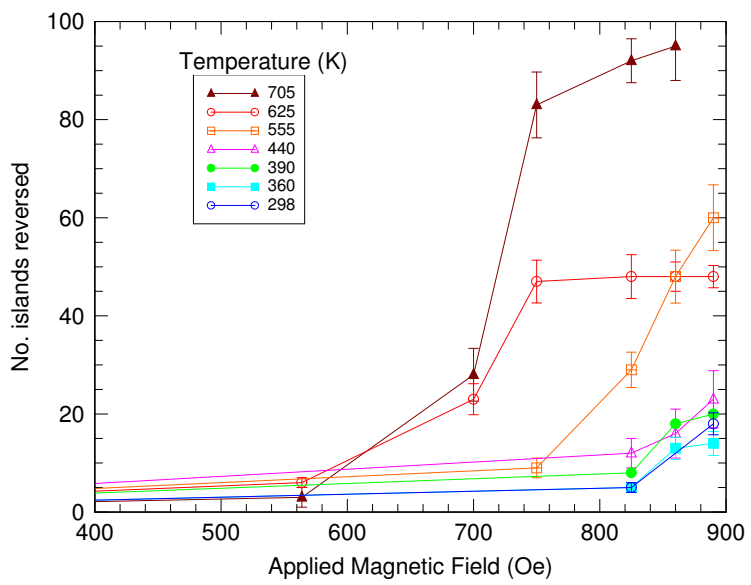

Figure 8. Number of flipped islands as the field $H$ is increased towards positive saturation for various different elevated array temperatures after negative saturation.

straight line fit to these data gives the observed flipping rate under these conditions, which was measured to be $r=0.12 \pm 0.02 \mathrm{~s}^{-1}$. Inserting this rate $r=1 / \tau$ into Eq. 1 yields a ratio $\Delta E / k_{\mathrm{B}} T=25.14 \pm 0.02$ at $T=300 \mathrm{~K}$ if we assume $f_{0}=10 \mathrm{GHz}$.

Here, the field is at $45^{\circ}$ to easy axis of all the islands. Under these circumstances, Harrell has shown that $n \sim 1.45,{ }^{32}$ close to the $n=3 / 2$ value of the Victora expansion. ${ }^{33}$ Assuming that $H_{0} \approx H_{\mathrm{c}}$, inserting $H / H_{\mathrm{c}}=0.9$ into Eq. 2 yields a an energy barrier $\Delta E$ that should be only $3.5 \%$ of the zero field value. This implies a ratio $\Delta E / k_{\mathrm{B}} T=43$ in this case. This value agrees reasonably well with the measured value, given the extreme sensitivity of the exponential function in Eq. 1 to its argument, although this agreement is to some extent fortuitous given the uncertainty over the true value of quantities such as $f_{0}$, and the fact that we have again neglected the inter-island coupling energy Ec. Assuming an activation volume smaller than the real island volume can also explain the discrepancy: the measured value of $\Delta E / k_{\mathrm{B}} T$ implies that the activation volume is approximately half of the true volume.

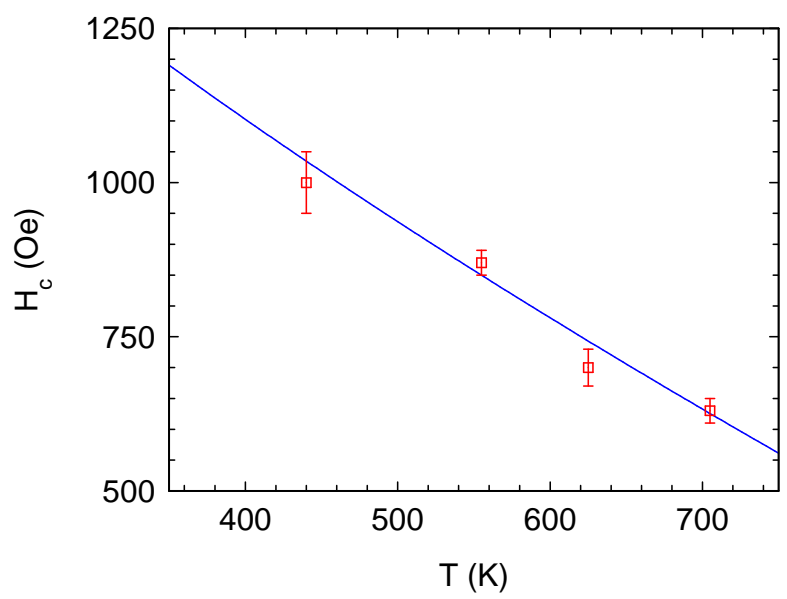

Figure 9. Coercive field $H_{\mathrm{c}}$ as a function of temperature $T$, derived from the data in Fig. 8. The line is a fit to the data as described in the text. 

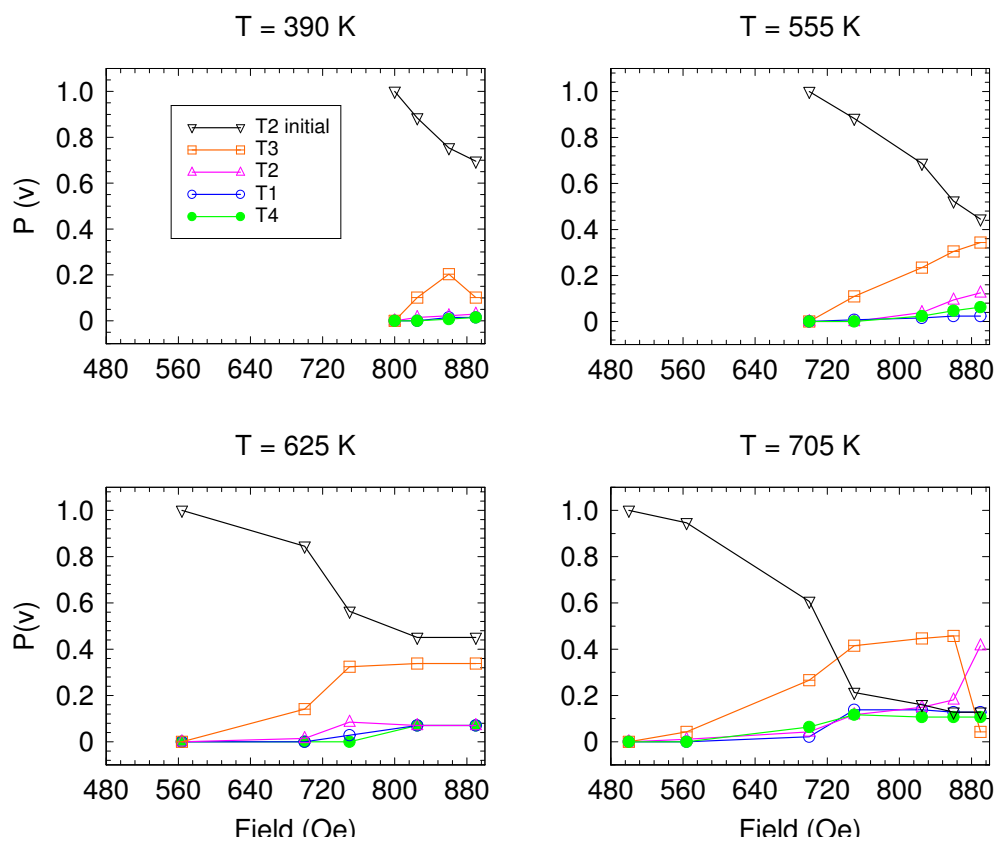

Figure 10. The population of different vertex types, $P(v)$, as a function of reversal field and shown for four temperatures; $390,555,625$, and $705 \mathrm{~K}$.

Having established that the barrier height can be tuned using applied field, we then investigated the effects of raising the temperature of the array. The samples were first saturated in a negative field along the array diagonal to set all the vertices into the same Type 2 configuration. The field was reversed to positive saturation, tracing out one branch of a hysteresis loop for the array. Fig. 8 shows the dependence of the hysteresis for this sample at different temperatures. These data show the field value at which the reversal is initiated but also how quickly it propagates as a function of temperature. It can be seen from the curves that there is little change between the data at room temperature and $440 \mathrm{~K}$, however, as the temperature was increased further the reversal began at lower field values indicating the onset of thermally activated reversal at these measurement timescales, $\sim 72 \mathrm{~s}$ (from $1.3 \mathrm{~s}$ and 5 averages for eleven field steps). There is also an increase in the number of islands which reversed within the area. This data is plotted as coercivity $H_{\mathrm{c}}$ as a function of temperature $T$ in Fig. 9 for those temperatures where a coercivity can be determined. It can be seen that there is a trend in decreasing coercivity of the array with temperature. We fitted this with Eq. 3, assuming $f_{0}=10 \mathrm{GHz}$ and $t=100 \mathrm{~s}$, and using the same value of $K$ as in the treatment of the flip rate data above. The Harrell value of $n=1.45$ was used. The result is shown in Fig. 9 as a line. The fit returned $H_{0}=2100 \pm 200$ Oe and $V=(1.9 \pm 0.1) \times 10^{5} \mathrm{~nm}$. The fact that $V$ is a little below the actual island volume shows once again that these islands do not reverse through an fully ideal coherent rotation mechanism.

This onset of thermally activated dynamics as the temperature rises is related to the pre-melting behavior of the macrospin system observed by Kapaklis et al. using a macroscopic magneto-optic measurements. ${ }^{20}$ Our use of a spatially resolving imaging method means that we are able to see exactly how the array begins to 'melt' and track the different vertex populations as this happens.

\subsection{Vertex populations}

Since we start with a known state, i.e. a background of Type 2 vertices, when we image the changes in contrast of certain islands we are also able to assign the resulting vertex type. Using this information we can map out the vertices in both field and temperature phase space, as shown in Fig. 10, where we show the fractional populations of the four different vertex types $P(v)$, where $v \in 1,2,3,4$ or $2_{\text {initial }}$. Type 2 vertices possess a dipole moment and here we have separated out the particular instance of Type 2 where that moment points along the initial saturation direction as a distinct vertex type, along the lines of the background Type 2 vertex defined by Nisoli 
et al. in their theory of effective temperature in a rotationally demagnetised square ice array. ${ }^{15}$ This allows us to distinguish between the saturated background and Nambu string defects that are carved in it by the field as it separates a monopole-antimonopole pair.

Our experiment extends the field-driven reversal measurements of Pollard et al. ${ }^{34}$ who studied square ices, or Mengotti et al., who studied kagomé ices, ${ }^{35}$ from the athermal regime to the onset of thermalization. The data are shown in Fig. 10 for four different measurement temperatures. At all four temperatures, the system begins with $P\left(2_{\text {initial }}\right)=1$ and all other populations at zero. As the field is swept, $P\left(2_{\text {initial }}\right)$ then drops as vertices are converted into other types by individual islands flipping their vertices. The fact that particular populations rise, indicates that there are preferred ways in which this can happen, due to the interisland coupling. We see a larger population of Type 3 excitations at higher temperatures, indicating an increased preference for nucleating monopole-antimonopole pairs over the propagation of strings through the sample. The latter would be signified by an increase in the values of $P(2)$, which is less pronounced. There is also an increase of the highest energy vertices, Type 4 , at the highest temperature, showing that the thermal energy is becoming sufficient to explore the whole of phase space. This can be contrasted with the findings of Pollard et al., who found that reversal predominantly occurs through the mechanism of string extension in the athermal limit. ${ }^{34}$

\section{IN SUMMARY}

We have been able to image directly the effect of increased temperature on the reversal of an ASI using a newly developed on-membrane heating device. We have shown increased dynamics and changes to the coercivity in the temeperature range $298-705 \mathrm{~K}$. We have also been able to track the individual vertex populations as a function of both field and temperature. As it is possible to apply magnetic fields during imaging, unlike electron based techniques, such as PEEM or Lorentz-TEM, M-TXM offers a unique opportunity to study thermal flipping behaviour with magnetic field. It also presents the possibility to push these frustrated nanomagnetic systems out of equilibrium whilst studying the resulting microstates.

\section{ACKNOWLEDGMENTS}

This work was supported by the EPSRC (grant numbers EP/J021482/1 and EP/I000933/1), the Science and Technology Facilities Council, and Diamond Light Source. Research carried out in part at the Center for Functional Nanomaterials, Brookhaven National Laboratory, which is supported by the U.S. Department of Energy, Office of Basic Energy Sciences, under Contract No. DE-AC02-98CH10886. X-ray microscopy studies were supported by the Director, Office of Science, Office of Basic Energy Sciences, of the U.S. Department of Energy under Contract No. DE-AC02-05CH11231. P. F. and M.-Y.I. acknowledge support by the Leading Foreign Research Institute Recruitment Program (Grant No. 2012K1A4A3053565) through the National Research Foundation of Korea (NRF) funded by the Ministry of Education, Science and Technology (MEST).

\section{REFERENCES}

1. C. Nisoli, R. Moessner, and P. Schiffer, "Colloquium: Artificial spin ice: Designing and imaging magnetic frustration," Rev. Mod. Phys. 85, p. 1473, 2013.

2. L. J. Heyderman and R. L. Stamps, "Artificial ferroic systems: novel functionality from structure, interactions and dynamics," J. Phys.: Cond. Matt. 25, p. 363201, 2013.

3. J. Cumings, L. J. Heyderman, C. H. Marrows, and R. L. Stamps, "Focus on artificial frustrated systems," New J. Phys. 16, p. 075016, 2014.

4. R. F. Wang, C. Nisoli, R. S. Freitas, J. Li, W. McConville, B. J. Cooley, M. S. Lund, N. Samarth, C. Leighton, V. H. Crespi, and P. Schiffer, "Artificial 'spin ice' in a geometrically frustrated lattice of nanoscale ferromagnetic islands," Nature 439, p. 303, 2006.

5. E. C. Stoner and E. P. Wohlfarth, "A mechanism of magnetic hysteresis in heterogeneous alloys," Phil. Trans. Roy. Soc. London A 240(826), p. 599, 1948.

6. S. T. Bramwell and M. J. P. Gingras, "Spin ice state in frustrated magnetic pyrochlore materials," Science 294, p. 1495, 2001. 
7. L. Pauling, "The structure and entropy of ice and of other crystals with some randomness of atomic arrangement," J. Am. Chem. Soc. 57, p. 2680, 1935.

8. A. P. Ramirez, A. Hayashi, R. J. Cava, R. Siddharthan, and B. S. Shastry, "Zero-point entropy in 'spin ice'," Nature 399, p. 333, 1999.

9. C. Castelnovo, R. Moessner, and S. L. Sondhi, "Magnetic monopoles in spin ice," Nature 451, p. 42, 2008.

10. J. D. Bernal and R. H. Fowler, "A theory of water and ionic solution, with particular reference to hydrogen and hydroxyl ions," J. Chem. Phys. 1, p. 515, 1933.

11. Y. Nambu, "Strings, monopoles, and gauge fields," Phys. Rev. D 10, p. 4262, 1974.

12. F. Wu, "Exactly soluble model of the ferroelectric phase transition in two dimensions," Phys. Rev. Lett. 18, pp. 605-607, 1967.

13. E. Lieb, "Exact solution of the problem of the entropy of two-dimensional ice," Phys. Rev. Lett. 18, pp. 692694, 1967.

14. C. Nisoli, R. Wang, J. Li, W. F. McConville, P. E. Lammert, P. Schiffer, and V. H. Crespi, "Ground state lost but degeneracy found: the effective thermodynamics of 'artificial spin ice'," Phys. Rev. Lett. 98, p. 217103, 2007.

15. C. Nisoli, J. Li, X. Ke, D. Garand, P. Schiffer, and V. H. Crespi, "Effective temperature in an interacting vertex system: Theory and experiment on artificial spin ice," Phys. Rev. Lett. 105, p. 047205, 2010.

16. P. E. Lammert, X. Ke, J. Li, C.Nisoli, D. M. Garand, V. H. Crespi, and P. Schiffer, "Direct entropy determination and application to artificial spin ice," Nature Physics 6, p. 786, 2010.

17. J. P. Morgan, J. Akerman, A. Stein, C. Phatak, R. M. L. Evans, S. Langridge, and C. H. Marrows, "Real and effective thermal equilibrium in artificial square spin ices," Phys. Rev. B 87, p. 024405, 2013.

18. X. Ke, J. Li, C. Nisoli, P. E. Lammert, W. McConville, R. F. Wang, V. H. Crespi, and P. Schiffer, "Energy minimization and ac demagnetization in a nanomagnet array," Phys. Rev. Lett. 101, p. 037205, 2008.

19. J. P. Morgan, A. Stein, S. Langridge, and C. H. Marrows, "Thermal ground-state ordering and elementary excitations in artificial magnetic square ice," Nature Phys. 7, p. 75, 2011.

20. V. Kapaklis, U. B. Arnalds, A. Harman-Clarke, E. T. Papaioannou, M. Karimipour, P. Korelis, A. Taroni, P. C. W. Holdsworth, S. T. Bramwell, and B. Hjörvarsson, "Melting artificial spin ice," New J. Phys. 14, p. 035009, 2012.

21. J. M. Porro, A.Bedoya-Pinto, A. Berger, and P. Vavassori, "Exploring thermally induced states in square artificial spin-ice arrays.," New J. Phys. 15, p. 055012, 2013.

22. S. Zhang, I. Gilbert, C. Nisoli, G.-W. Chern, M. J. Erickson, L. O’Brien, C. Leighton, P. E. Lammert, V. H. Crespi, and P. Schiffer, "Crystallites of magnetic charges in artificial spin ice," Nature 500, p. 553, 2013.

23. A. Farhan, P. M. Derlet, A. Kleibert, A. Balan, R. V. Chopdekar, M. Wyss, L. Anghinolfi, F. Nolting, and L. J. Heyderman, "Exploring hyper-cubic energy landscapes in thermally active finite spin-ice systems," Nature Phys. 9, p. 375, 2013.

24. V. Kapaklis, U. B. Arnalds, A. Farhan, R. V. C. A. Balan, A. Scholl, L. J. Heyderman, and B. Hjörvarsson, "Thermal fluctuations in artificial spin ice," Nature Nanotech. 9, p. 514, 2014.

25. A. Farhan, P. M. Derlet, A. Kleibert, A. Balan, R. V. Chopdekar, M. Wyss, J. Perron, A. Scholl, F. Nolting, and L. J. Heyderman, "Direct observation of thermal relaxation in artificial spin ice," Phys. Rev. Lett. 111, p. $057204,2013$.

26. D. A. Allwood, G. Xiong, M. D. Cooke, and R. P. Cowburn, "Magneto-optical Kerr effect analysis of magnetic nanostructures," J. Phys. D: Appl. Phys. 36, p. 2175, 2003.

27. C. P. Bean and J. D. Livingston, "Superparamagnetism," J. Appl. Phys. 30, p. S120, 1959.

28. M. P. Sharrock, "Time dependence of switching fields in magnetic recording media (invited)," J. Appl. Phys. 76, p. 6413, 1994.

29. A. Aharoni, "Demagnetizing factors for rectangular ferromagnetic prisms," J. Appl. Phys. 83, p. 3442, 1998.

30. C. Phatak, A. K. Petford-Long, O. Heinonen, M. Tanase, and M. De Graef, "Nanoscale structure of magnetic induction at monopole defects in artificial spin-ice lattices," Phys. Rev. B 83, p. 174431, 2011.

31. P. Fischer, D.-H. Kim, W. Chao, J. A. Liddle, E. H. Anderson, and D. T. Attwood, "Soft X-ray microscopy of nanomagnetism," Materials Today 9, p. 26, 2006. 
32. J. W. Harrell, "Orientation dependence of the dynamic coercivity of Stoner-Wohlfarth particles," IEEE Trans. Magn. 37, p. 533, 2001.

33. R. H. Victora, "Predicted time dependence of the switching field for magnetic materials," Phys. Rev. Lett. 63, p. $457,1989$.

34. S. D. Pollard, V. Volkov, and Y. Zhu, "Propagation of magnetic charge monopoles and Dirac flux strings in an artificial spin-ice lattice," Phys. Rev. B 85, p. 180402, 2012.

35. E. Mengotti, L. J. Heyderman, A. F. Rodríguez, F. Nolting, R. V. Hügli, and H.-B. Braun, "Real-space observation of emergent magnetic monopoles and associated Dirac strings in artificial kagome spin ice," Nature Phys. 7, p. 68, 2011. 